\title{
Der Schlaf bei Anorexia nervosa, Bulimia nervosa und depressiven Erkrankungen: Eine polysomnographische Vergleichsstudie
}

\author{
Ch. Lauer ${ }^{1,2}$, J.Ch. Krieg ${ }^{1.2}$, J. Zulley ${ }^{2}$, D. Riemann ${ }^{3}$, M. Berger ${ }^{3}$ \\ 1 Psychiatrische Universitätsklinik, Freiburg \\ 2 Max-Planck-Institut für Psychiatrie, München \\ 3 Zentralinstitut für seelische Gesundheit, Mannheim
}

\section{Sleep in Anorexia Nervosa, Bulimia Nervosa and Depressive Disorders}

All-night EEG sleep in 20 anorexics, 10 bulimics, 10 endogenous depressives, and in 10 healthy subjects (all age matched) was compared. In addition, the REM sleep-induction-test was performed in 12 patients with an eating disorder, 7 depressives, and 12 controls by application of the cholinergic agent RS 86. During baseline night, EEG-sleep parameters, especially REM latency, did not differ between the patients and the controls, except for the phasic components of REM sleep (REM density) that were increased in the depressive patients. The frequency of shortened REM latencies, however, was significantly higher in the depressed patients. These observations indicate that in some of the young depressives the disturbance of the REM sleep regulating transmitter system is already present to a similar degree as it is assumed in elderly depressives. After the application of RS 86, REM latency was shortened in all groups under investigation. However, the REM sleep inducing effect of RS 86 was significantly more pronounced in the depressives when compared with both the eating disorder patients and the controls. In the latter two samples, the shortening of REM latency was similar. Furthermore, the eating disorder patients with a concomitant major depression reacted similar to RS 86 as the non-depressed eating disorder patients and the control subjects.

Whereas baseline EEG-sleep did not differ significantly among eating disorder patients, young depressives, and healthy subjects, the REM sleep inducing effect of the cholinergic agent RS 86 clearly distinguished between the depressives and both the patients suffering from eating disorders and the controls. These results are not in line with the assumption of a biological link between eating disorder and depression, but support those models supposing the depressive symptomatology in eating disorder as a secondary phenomenon.

\section{Zusammenfassung}

Zwanzig Patienten mit Anorexia nervosa, 10 Patienten mit Bulimia nervosa, 10 endogen Depressive und 10 Gesunde wurden polysomnographisch untersucht. Weiterhin wurde bei 12 Patienten mit einer EBstörung, bei 7 Depressiven und 12 Kontrollen der REM-Schlaf-Induktions-Test mit dem cholinergen Agonisten RS 86 durchgeführt.

Mit Ausnahme der phasischen REM-SchlafKomponenten (REM-Dichte), die bei den Depressiven im Vergleich zu den anderen Gruppen erhöht waren, konnten während der Baseline-Nächte keine Gruppen-Unterschiede in den Schlaf-Parametern beobachtet werden. Obwohl dies auch für die mittlere REM-Latenz der Fall war, wurde bei den Depressiven häufiger eine verkürzte REM-Latenz gefunden; dies spricht dafür, $\mathrm{da} ß$ bei einigen dieser jungen Depressiven bereits eine Störung des den REM-Schlaf regulierenden TransmitterSystems vorliegt. Nach der Applikation von RS 86 zeigte sich bei den jungen depressiven Patienten eine vollständige Manifestation der für ältere Depressive typischen REM-Schlaf-Dysregulation. Zwar konnte auch bei den Patienten mit einer EBstörung und den gesunden Probanden ein REM-Schlaf-induzierender Effekt von RS 86 nachgewiesen werden, dieser war jedoch in beiden Gruppen im Vergleich zu den Depressiven deutlich schwächer ausgeprägt.

Diese Resultate sprechen gegen eine biologische Assoziation von Eßstörung und Depression. Sie stützen vielmehr jene Modelle, die die bei anorektischen und bulimischen Patienten häufig zu beobachtende depressive Symptomatik als sekundäres Phänomen der Eßstörung ansehen.

\section{Einführung}

Die nosologische Einordnung der EBstörungen war anfänglich umstritten. So rechnte z. B. Kraepelin (28) die

Fortschr. Neurol. Psychiat. 57(1989) 403-410

(C) Georg Thieme Verlag Stuttgart - New York
Magersucht zum Spektrum der affektiven Erkrankungen, Binswanger (5) hingegen ordnete sie der Schizophrenie zu. Es setzte sich dann jedoch die Überzeugung durch, $\mathrm{da} B$ es sich bei der Anorexia nervosa und Bulimia nervosa um eigenständige Krankheitsbilder handelt. Aufgrund psychopathologischer und neuroendokrinologischer Studien, sowie Verlaufs- und Familien-Untersuchungen (Übersichten bei $18,46,47$ ) ist in letzter 
Zeit die Diskussion um die nosologische Zuordnung der Eßstörung zur Depression erneut aufgeflammt. Einige Autoren betrachten die Eßstörung als Variante einer primär depressiven Erkrankung, wobei die EB- und gewichtsbezogenen Symptome lediglich als sekundäre Begleitphänomene gewertet werden (8, 21, 25). In anderen Modellen wird die dysphorische Verstimmung anorektischer Patienten auf mangelernährungsbedingte physiologische und psychologische Veränderungen zurückgeführt $(7,9)$, bzw. bei der Bulimia nervosa als Konsequenz des Kontrollverlustes über das Eßverhalten und den daraus resultierenden Insuffizienzgefühlen interpretiert (12).

Aus dem Bereich der Schlafforschung kamen bislang kaum Beiträge zu dieser Diskussion. Dies ist um so überraschender, da bestimmte Veränderungen im Schlaf depressiver Patienten, besonders eine verkürzte Latenzzeit der ersten REMPeriode und eine erhöhte Augenbewegungsaktivität während des REM-Schlafes, als charakteristische biologische Normabweichungen der Depression angesehen werden (Übersicht bei 17, 29).

Die erste polysomnographische Untersuchung anorektischer Patienten führten Crisp und Mitarbeiter (10) durch. Sie beobachteten eine gestörte Schlaf-Kontinuität (Einund Durchschlaf-Störungen, frühmorgendliches Erwachen) und einen reduzierten Tiefschlaf-Anteil. Diese Ergebnisse konnten von anderen Arbeitsgruppen repliziert werden $(31,33,38)$. In drei Studien wurde weiterhin bei einigen anorektischen Patienten eine verkürzte REM-Latenz beobachtet $(26,38,49)$, wobei die meisten dieser Patienten gleichzeitig die DSM-III Kriterien (27) einer ,major depression“ erfüllten.

Das Schlafmuster von bulimischen Patienten hingegen war weitgehend als nicht von dem gesunder Kontrollen differenzierbar beschrieben worden $(22,33,49)$. Walsh und Kollegen (49) berichteten jedoch, daß depressive Bulimie-Patienten eine gegenüber den nicht depressiven Bulimien tendenziell verkürzte REM-Latenz aufwiesen. Auch Weilburg und Mitarbeiter (50) beobachteten bei bulimischen Patienten eine kurze Latenz bis zur ersten REM-,,ähnlichen"-Periode (diese REM-Periode war jedoch sehr kurz und es traten keine schnellen Augenbewegungen auf). Die bisher einzige Studie, in welcher die Schlafstruktur von Bulimie-Patienten mit der Depressiver verglichen wurde (22), erbrachte eine bessere Schlafgüte und eine niedrigere REM-Dichte bei den bulimischen Patienten.

Insbesonders die bei einigen eßgestörten $\mathrm{Pa}$ tienten, welche zudem die formalen diagnostischen Kriterien einer ,,major depression“ erfüllten, beobachtete verkürzte REMLatenz scheint somit die Annahme einer biologischen Assoziation von EBstörung und Depression zu stützen.

Eine weitere Möglichkeit, um die in der Diskussion stehende Thematik zu untersuchen, bietet der cholinerge REM-Schlaf-Induktion-Test (RIT). Das Rational dieses Testes basiert auf der von Janowsky und Mitarbeitern (23) formulierten noradrenerg-cholinergen Imbalance-Theorie der Depression und auf dem neurochemischen Schlaf-Regulations-Modell (19, 36). In diesem Modell wird angenommen, daß REM-Schlaf von aminergen Neuronenverbänden gehemmt, von cholinergen Neuronenverbänden jedoch stimuliert wird. Der zyklische Wechsel von NonREM- und REM-Schlaf wird durch eine reziproke Interaktion dieser Transmittersysteme generiert. In Anlehnung an die Imbalance-Theorie der Depression werden die für die Depression typischen REM-Schlaf-Veränderungen auf ein relatives Übergewicht des cholinergen über das aminerge System interpretiert (siehe Übersicht bei 2). Gillin und Kollegen (16) konnten demonstrieren, da $B$ das den REM-Schlaf regulierende System bei Depressiven deutlich empfindlicher auf einen cholinergen Stimulus wie etwa Physostigmin oder Arecolin reagierte, als dies bei Gesunden der Fall war; mit anderen Worten, nach Applikation eines Cholinomimetikums war die REM-Latenz der Depressiven erheblich mehr verkürzt. Bei anorektischen Patienten wurde der RIT bislang nur von Sitaram und Mitarbeitern (44) durchgeführt. Sie berichteten, daß $66 \%$ der anorektischen Patienten mit der Zusatzdiagnose einer ,,major depression" und $71 \%$ der untersuchten Depressiven als ,Responder" auf die Gabe von Arecolin eingestuft werden konnten. Bei nicht depressiven Anorexie-Patienten und Gesunden war die Anzahl der Responder gering (12\%, bzw. 25\%). Auch diese Resultate scheinen für eine biologische Assoziation von EBstörung und Depression zu sprechen.

Vor kurzem führte Spiegel (45) ein weiteres Cholinomimetikum, RS 86, in die Schlafforschung ein. Das Spiropiperidyl-Derivat RS 86 weist gegenüber Physostigmin und Arecolin deutliche methodische Vorteile auf. Es hat im Gegensatz zu den beiden anderen cholinergen Substanzen nur geringe periphere Nebenwirkungen, und kann aufgrund seiner langen Halbwertszeit von ca. 8 Stunden oral appliziert werden. Physostigmin und Arecolin hingegen, deren Halbwertszeit bei etwa 30 Minuten liegt, müssen während des Schlafes intravenös appliziert werden. Spiegel (45) demonstrierte, daß nach der Gabe von $1.5 \mathrm{mg}$ RS 86 die REM-Latenz Gesunder verkürzt war. Diese Ergebnisse konnten von unserer Arbeitsgruppe nicht nur repliziert werden, sondern es wurde weiterhin gezeigt, daß dieser REM-Schlaf induzierende Effekt von RS 86 bei Depressiven wesentlich stärker ausgeprägt war als bei altersparallelisierten Kontrollen $(3,4,41)$.

Die Fragestellungen, welche anhand der vorliegenden Studie abgeklärt werden sollen, lauten:

- Lassen sich im Schlaf anorektischer und bulimischer Patienten Abweichungen von dem Schlafmuster gesunder Kontrollen finden, welche auch für depressive Patienten beschrieben werden?

- Sind aufgrund des cholinergen REM-Induktions-Test Hinweise erkennbar, welche für eine biologische Assoziation von EBstörung und Depression sprechen?

\section{Methodik \\ Stichproben}

Es wurden 20 Patienten (mittleres Alter: $21 \mathrm{~J}$; mittleres Gewicht: $70 \%$ IBW, ermittelt anhand der Tabellen in 37), welche die Feighner-Kriterien einer Anorexia nervosa (13) erfüllten, sowie 10 Patienten mit einer Bulimia nervosa (diagnostiziert nach 42; mittl. Alter: $23 \mathrm{~J}$; mittl. Gewicht: $98 \%$ IBW) untersucht (weitere Charakteristika dieser Patienten sind in Tabelle 1 zusammengefaßt).

Weiterhin wurden 10 depressive Patienten untersucht, deren Altersverteilung so eng wie möglich an die der eßgestörten Patienten angeglichen war (mittl. Alter: 23 J.; mittl. Gewicht: $103 \%$ IBW). Nach der Internationalen Klassifikation der Krankheiten, 9. Revision (ICD-9; 11) waren diese Patienten 
Tab. 1 Charakteristika der Stichproben

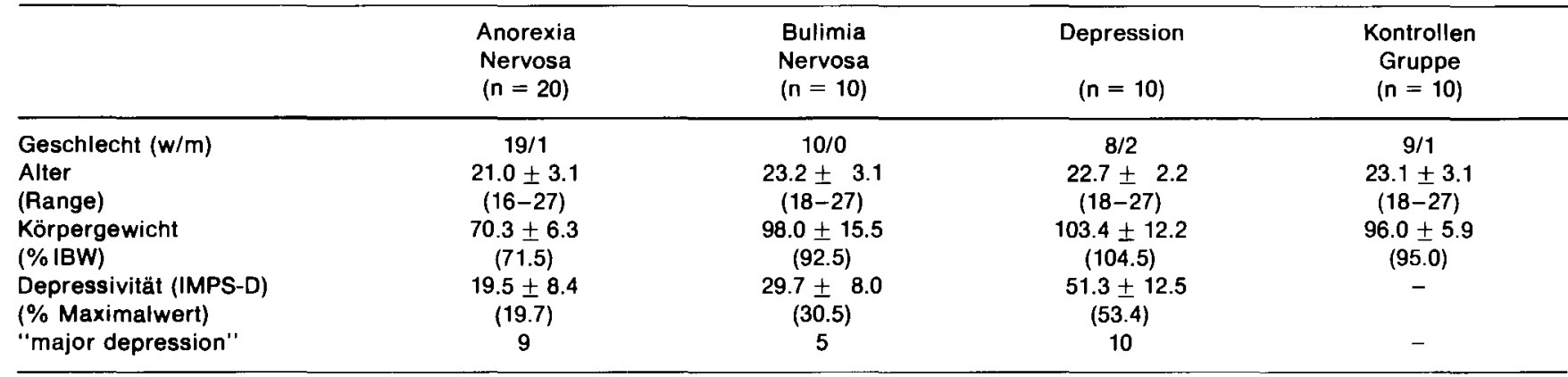

als endogen diagnostiziert worden. Fünf Patienten zeigten einen monopolaren, 5 einen bipolaren Verlauf der affektiven Psychose. Jeder dieser Patienten erfüllte auch die DSM-III Kriterien einer „major depression“, 6 von ihnen auch die des Subtypes „Melancholia“. Bei der Auswahl dieser Patienten wurde darauf geachtet, daß sie keine Anzeichen einer Anorexia nervosa oder Bulimia nervosa aufwiesen.

Die DSM-III-Diagnose einer ,,major depression" wurde mittels der deutschsprachigen Version des ,Composit International Diagnostic Interview“ (CIDI; 43) gestellt. Der Schweregrad der depressiven Symptomatik wurde anhand der depressionsrelevanten Items der ,Inpatient Multidimensional Psychiatric Scale" (IMPS; Subskalen: Depressive Verstimmtheit (ANX), Verlangsamung/Apathie (RTD), Erschöpftheit/ Vitalstörung (IMP); 34) erhoben.

Alle Patienten wurden stationär auf der offenen Station der psychiatrischen Abteilung des Max-Planck-Institutes für Psychiatrie, München, untersucht und behandelt.

Die 10 gesunden Kontrollen waren in ihrer Alters- und Geschlechtsverteilung ebenfalls an die der eßgestörten Patienten angeglichen (mittl. Alter: $23 \mathrm{~J}$; mittl. Gewicht: $98 \%$ IBW). Mit Hilfe eines halbstrukturierten Interviews wurde eine eigene, sowie eine familiäre psychiatrische Erkrankung ausgeschlossen. Weiterhin hatten die Kontrollen ein „ungestörtes“ Eßverhalten aufzuweisen (kein Diätieren, keine Nahrungsselektion, eine normale Hauptmahlzeit pro Tag).

\section{Schlaf}

Zum Zeitpunkt der Polysomnographie betrug der medikationsfreie Zeitraum bei den eßgestörten Patienten mindestens 3 Monate, bei 5 der Depressiven mindestens 6 Monate. Die weiteren 5 depressiven Patienten waren zwischen 8 und 21 Tagen frei von psychoaktiver Medikation. Die Patienten schliefen für drei, die Kontrollen für mindestens zwei aufeinanderfolgende Nächte im Schlaflabor. Die Polysomnographie (EEG, EOG, EMG) begann um 23:15 Uhr (Licht aus) und endete um 6:30 Uhr (Licht an). Die Aufzeichnungen der 3. Nacht, bzw. bei den Kontrollen der 2. Nacht, wurden visuell nach Standardkriterien (40) ausgewertet. Die Definition der analysierten Schlafparameter wurde bereits beschrieben (41).

\section{Der cholinerge REM-Schlaf-Induktions-Test (RIT)}

Zusätzlich zu der Baseline-Polysomnographie nahmen 6 anorektische Patienten nach Erreichen von minde- stens $80 \%$ des IWB (Alter: $22.5 \pm 3.1 \mathrm{~J}$.; Gewicht: $85.5 \pm 5.1 \%$ des IBW; ,major depression“" $\mathrm{n}=2$ ) und 6 bulimische Patienten (Alter: $21.5 \pm 2.7 \mathrm{~J}$; Gewicht: $91.5 \pm 7.7 \%$ des IBW; ,major depression": $n=4$ ) an dem RIT teil. Der Schweregrad der Depression bei diesen 12 Patienten betrug im Mittel $24 \%$ des Maximalwertes der depressionsrelevanten Items der IMPSSkala (IMPS-D).

Die Kontrollpersonen $\quad(\mathbf{n}=12 ; \quad$ Alter: $23.3 \pm 2.8 \mathrm{~J}$.) und die depressiven Patienten $(\mathrm{n}=7$; Alter: $25.7 \pm 6.8 \mathrm{~J}$.) wurden einer größeren Stichprobe entnommen (4, 41). Kriterium hierfür war ein Alter $<35$ Jahren, um die Altersverteilung dieser beiden Gruppen so eng wie möglich an die der eßgestörten Patienten anzugleichen. Drei der depressiven Patienten waren als endogen, 4 als neurotisch depressiv klassifiziert worden (nach ICD-9). Jeder dieser Patienten erfüllte die DSMIII Kriterien einer ,major depression“, 4 davon auch die des Subtypes „Melancholia“. Die Patienten und Kontrollen nahmen um 22:00 Uhr in randomisierter Reihenfolge ein PlaceboPräparat oder $1.5 \mathrm{mg}$ RS 86 in Kapselform ein. Ableitung und Auswertung des Schlafes erfolgte wie oben beschrieben. Die Teilnehmer wurden vor Beginn des RIT auf eventuelle Nebenwirkungen von RS 86 hingewiesen und um ihre schriftliche Einverständniserklärung gebeten.

\section{Statistische Methoden}

Als Maße der deskriptiven Statistik wurden Mittelwert und Standardabweichung bestimmt. Als Signifikanztest kam die Varianzanalyse (ANOVA, MANOVA), sowie der $T$-Test (bei inhomogenen Varianzen in der nach Welch korrigierten Form) zur Anwendung. Korrelative Zusammenhänge wurden mittels des Spearman-Rang-Korrelations-Koeffizienten bestimmt. Alle Verfahren gingen von einem $\alpha=0.5$ aus und das Signifikanzniveau wurde auf $5 \%$ festgelegt.

\section{Ergebnisse}

Die Alters- und Geschlechtsverteilung der beiden Gruppen mit Eßstörung, der Depressiven und der Kontrollen war vergleichbar. Wie erwartet, wiesen die anorektischen $\mathrm{Pa}$ tienten ein signifikant geringeres Körpergewicht (\% IBW) auf als die übrigen drei Stichproben $(F=34.71, \mathrm{df}=3, \mathrm{p}<0.001)$. Ein signifikanter Gruppeneffekt zeigte sich für den Schweregrad der depressiven Symptomatik $(F=37,54, \mathrm{df}=2, \mathrm{p}<0.001)$, welcher bei den anorektischen Patienten niedriger war als bei den Bulimie-Patienten ( $\mathrm{T}=3.17$, df $=28, \mathrm{p}<0.01$ ) und den Depressiven ( $\mathrm{T}=8.30, \mathrm{df}=28, \mathrm{p}<0.001)$. Gegenüber den Depressiven erwiesen sich auch die bulimischen Patienten als geringer depressiv $(\mathrm{T}=4.61, \mathrm{df}=18, \mathrm{p}<0.01)$. In der Gruppe der 
Tab. 2 Statistik der Schlaf-Parameter der Patienten mit Anorexia nervosa $(n=20)$, Bulimia nervosa $(n=10)$, Depressiven $(n=10)$ und Kontrollpersonen $(n=10)$.

\begin{tabular}{|c|c|c|c|c|c|c|}
\hline & & $\begin{array}{l}\text { Anorexia } \\
\text { Nervosa }\end{array}$ & $\begin{array}{l}\text { Bulimia } \\
\text { Nervosa }\end{array}$ & Depression & $\begin{array}{l}\text { Kontrollen } \\
\text { Gruppe }\end{array}$ & $\begin{array}{c}\text { Anova } \\
F \mathrm{df}=3 \mathrm{p}\end{array}$ \\
\hline Schlaf-Periode & (SPT; min) & $408.8 \pm 25.5$ & $408.3 \pm 32.7$ & $416.5 \pm 33.2$ & $413.7 \pm 17.0$ & 0.23 \\
\hline Schlafgüte & $(\%)$ & $90.2 \pm 6.8$ & $87.9 \pm 6.7$ & $89.2 \pm 9.2$ & $93.2 \pm 4.2$ & 1.11 \\
\hline Einschlaf-Latenz & $(\min )$ & $14.9 \pm 12.9$ & $22.1 \pm 17.6$ & $21.1 \pm 15.9$ & $19.0 \pm 10.6$ & 0.75 \\
\hline nächtliche Wachzeit & $(\min )$ & $15.9 \pm 18.4$ & $21.2 \pm 23.7$ & $24.3 \pm 45.0$ & $7.8 \pm 11.0$ & 0.79 \\
\hline frühmorgendl. Erwachen & $(\min )$ & $10.8 \pm 16.8$ & $9.6 \pm 19.0$ & $3.9 \pm 7.1$ & $2.4 \pm 4.8$ & 1.08 \\
\hline Schlafstadium 1 & (\%SPT) & $6.0 \pm 3.8$ & $8.4 \pm 4.8$ & $6.9 \pm 4.0$ & $5.9 \pm 1.8$ & 0.98 \\
\hline Schlafstadium & (\%SPT) & $50.1 \pm 9.3$ & $46.2 \pm 12.9$ & $43.3 \pm 4.8$ & $52.1 \pm 5.7$ & 2.07 \\
\hline Tiefschlaf & (\%SPT) & $20.2 \pm 10.1$ & $18.5 \pm 9.1$ & $21.2 \pm 8.6$ & $19.6 \pm 7.6$ & 0.15 \\
\hline REM-Schlaf & (\%SPT) & $19.2 \pm 6.5$ & $20.4 \pm 6.1$ & $22.6 \pm 8.5$ & $19.4 \pm 3.7$ & 0.67 \\
\hline REM-Latenz & $(\min )$ & $69.4 \pm 26.1$ & $94.0 \pm 79.1$ & $56.4 \pm 29.8$ & $72.0 \pm 31.5$ & 1.32 \\
\hline REM-Schlaf & $(\min )$ & $78.7 \pm 27.2$ & $82.5 \pm 19.8$ & $93.7 \pm 36.2$ & $80.1 \pm 14.4$ & 0.78 \\
\hline Dauer 1. REM-Periode & $(\min )$ & $14.7 \pm 14.9$ & $19.3 \pm 17.1$ & $17.8 \pm 19.5$ & $14.6 \pm 8.6$ & 0.27 \\
\hline 1. REM-Dichte & $(\%)$ & $19.7 \pm 7.4$ & $20.3 \pm 12.0$ & $31.1 \pm 13.0$ & $22.4 \pm 8.5$ & $3.22<0.05$ \\
\hline mittlere REM-Dichte & $(\%)$ & $25.8 \pm 8.4$ & $28.0 \pm 9.8$ & $41.2 \pm 9.7$ & $32.2 \pm 10.5$ & $6.37<0.001$ \\
\hline
\end{tabular}

anorektischen und bulimischen Patienten war die Verteilung der Patienten, welche die DSM-III Kriterien einer ,major depression" erfüllten, identisch.

In Tabelle 2 sind für die analysierten Schlafparameter die Resultate der deskriptiven Statistik, sowie der ANOVA dargestellt. Weder für die Variablen der Schlaf-Kontinuität, noch der Schlaf-Architektur konnten statistisch abzusichernde Gruppen-Effekte gefunden werden.

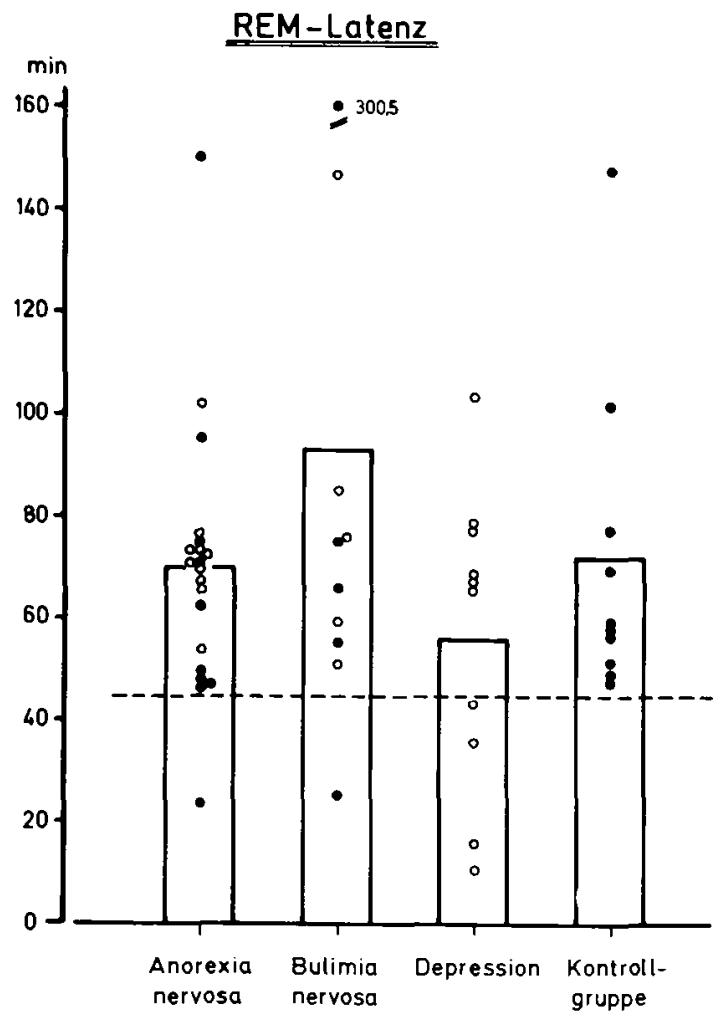

Abb. 1 REM-Latenz der Baseline-Nächte von Patienten mit Anorexia nervosa $(n=20)$, Patienten mit Bulimia nervosa $(n=10)$, Depressiven $(n=10)$ und gesunden Kontrollen $(n=10)$. Die Säulen zeigen den Mittelwert. Die offenen Kreise präsentieren die Patienten, welche die DSM-III-Kriterien einer „,major depression" erfüllten.
Entgegen den Erwartungen war dies auch für die tonischen REM-Schlaf-Parameter der Fall (REM-Latenz, Anteil des REM-Schlafes; Tab. 2; Abb. 1), obwohl die Depressiven im Mittel eine deutlich kürzere REM-Latenz als die übrigen drei Gruppen zeigten. Ein Vergleich der Häufigkeit von verkürzten REM-Latenzen ( < 45 Minuten) ergab, da 3 die Depressiven signifikant öfter eine verkürzte REM-Latenz aufwiesen als dies in den drei anderen Stichproben gefunden wurde (Chi-Qua$\mathrm{drat}=18.4, \mathrm{df}=3, \mathrm{p}<0.001)$. An dieser Stelle sei darauf hingewiesen, daß die mittlere REM-Latenz der bulimischen Patienten durch einen „Ausreißer"-Wert von 300.5 Minuten artifiziell erhöht war. Die phasischen Komponenten des REM-Schlafes (REM-Dichte) waren bei den depressiven Patienten im Vergleich zu den Patienten mit Anorexia nervosa und Bulimia nervosa signifikant erhöht. Die REM-Dichte der Kontrollpersonen, welche sich nicht von der der anorektischen und bulimischen Patienten unterschied, war gegenüber der bei Depressiven tendenziell erniedrigt $(\mathrm{T}=1.98, \mathrm{df}=18, \mathrm{p}<0.07)$.

Ein Vergleich der eßgestörten Patienten, die die DSM-III-Kriterien einer ,,major depression" erfüllten, mit jcnen, die keine ,major depression" aufwiesen, erbrachte für keine der analysierten Schlafvariablen einen statistisch abzusichernden Unterschied.

In keiner der 4 Gruppen ergaben sich signifikante Korrelationen zwischen dem Körpergewicht ( $\%$ IBW), der Depressivität (IMPS-D) und den Schlaf-Parametern. Für die Depressiven und auch für die bulimischen Patienten wurde jedoch eine signifikant negative Korrelation von Alter und REMLatenz gefunden $(r=-.65, p<0.05 ; r=-.66, p<0.05)$.

\section{REM-Schlaf-Induktions-Test (RIT)}

Bei keinem der Patienten und der Kontrollen traten nach Einnahme von $1.5 \mathrm{mg}$ RS 86 merkliche Nebenwirkungen auf. In Tabelle 3 sind die Ergebnisse der deskriptiven Statistik der Schlafvariablen unter Plazebo und nach RS 86, sowie der Signifikanzprüfung zusammengefaßt. Ein deutlicher, jedoch nicht signifikanter Gruppen-Effekt zeigte sich lediglich für die REM-Dichte der 1. REM-Periode. Nach Applikation von RS 86(,Treatment"-Effekt) war die Einschlaf-Latenz verlängert und der Anteil des Tiefschlafes war reduziert. Der Anteil des 
Tab. 3 Schlatvariablen der Plazebo- und RS 86-Nacht von den Patienten mit einer EGstörung $(n=12)$, Depression $(n=7)$, und Kontrolipersonen $(n=12)$.

\begin{tabular}{|c|c|c|c|c|c|c|c|}
\hline \multirow{2}{*}{\multicolumn{2}{|c|}{ EB-Störung }} & \multicolumn{2}{|c|}{ Depression } & \multicolumn{2}{|c|}{ Kontroll-Gruppe } & \multirow[b]{2}{*}{ Plazebo } & \multirow[b]{2}{*}{ RS 86} \\
\hline & & Plazebo & RS 86 & Plazebo & RS 86 & & \\
\hline $\begin{array}{l}\text { Schlaf-Periode } \\
\text { Einschlaf-Latenz } \\
\text { Intermitt. Wach } \\
\text { Schlafgüte }\end{array}$ & $\begin{array}{r}(\min ) \\
(\min ) \\
(\min ) \\
(\%)\end{array}$ & $\begin{array}{r}383.3 \pm 49.3 \\
14.9 \pm 12.4 \\
10.7 \pm 17.7 \\
87.5 \pm 10.9\end{array}$ & $\begin{array}{r}398.2 \pm 23.6 \\
18.9 \pm 11.0 \\
24.2 \pm 26.5 \\
87.3 \pm 11.3\end{array}$ & $\begin{array}{r}420.6 \pm 33.3 \\
13.2 \pm 8.2 \\
36.4 \pm 69.6 \\
86.7 \pm 21.0\end{array}$ & $\begin{array}{l}401.1 \pm 32.0 \\
34.1 \pm 12.8^{* * *} \\
21.4 \pm 24.9 \\
85.4 \pm 10.4\end{array}$ & $\begin{array}{r}417.0 \pm 16.2 \\
13.7 \pm 10.4 \\
10.4 \pm 9.4 \\
94.0 \pm 4.0\end{array}$ & $\begin{array}{r}410.7 \pm 18.5 \\
16.6 \pm 7.5 \\
13.5 \pm 10.4 \\
92.3 \pm 3.3\end{array}$ \\
\hline $\begin{array}{l}\text { Tiefschlaf } \\
\text { REM-Schlaf }\end{array}$ & $\begin{array}{l}(\%) \\
(\%)\end{array}$ & $\begin{array}{l}22.7 \pm 8.1 \\
20.3 \pm 4.2\end{array}$ & $\begin{array}{l}10.9 \pm 9.8^{* * *} \\
22.0 \pm 5.2\end{array}$ & $\begin{array}{l}13.8 \pm 8.7 \\
20.5 \pm 2.9\end{array}$ & $\begin{array}{r}9.9 \pm 7.7 \\
23.5 \pm 5.5^{*}\end{array}$ & $\begin{array}{l}19.6 \pm 7.7 \\
18.7 \pm 2.4\end{array}$ & $\begin{array}{l}14.5 \pm 7.7^{*} \\
21.6 \pm 3.1^{*}\end{array}$ \\
\hline
\end{tabular}

Hauptkomponenten-Analyse (MeBwiederholungs-Design): * $p<0.05 \quad$ " $p<0.01 \quad$ *** $p<0.001$

Ergebnisse der MANOVA hinsichtlich des Einflusses von RS 86 auf den Schlat der drei untersuchten Gruppen

\begin{tabular}{|c|c|c|c|c|c|}
\hline \multirow[b]{2}{*}{$\begin{array}{l}\text { Schlaf-Periode } \\
\text { Einschlaf-Latenz } \\
\text { Intermitt. Wach } \\
\text { Schlafgüte }\end{array}$} & $\begin{array}{l}\text { Diagnosen } \\
F \text { df }=2 p\end{array}$ & $\begin{array}{l}\text { anova: } \\
\text { "Treat } \\
\text { F df = }\end{array}$ & $\begin{array}{l}\text { Haupteff } \\
\text { iment" } \\
=1 \mathrm{p}\end{array}$ & $\begin{array}{l}\text { fekte } \\
\text { Interal } \\
\text { F df }=\end{array}$ & $\begin{array}{l}\text { ktion } \\
2 p\end{array}$ \\
\hline & $\begin{array}{r}(\min ) 2.43 \\
(\min ) 2.44 \\
(\min ) 0.99 \\
(\%) 1.52\end{array}$ & $\begin{array}{r}0.49 \\
14.73 \\
0.01 \\
0.54\end{array}$ & .001 & $\begin{array}{l}3.69 \\
4.88 \\
2.17 \\
0.11\end{array}$ & $\begin{array}{l}.038 \\
.015\end{array}$ \\
\hline $\begin{array}{l}\text { Tiefschlaf } \\
\text { REM-Schlaf }\end{array}$ & $\begin{array}{l}(\%) 1.24 \\
(\%) 0.66\end{array}$ & $\begin{array}{l}25.84 \\
12.43\end{array}$ & $\begin{array}{r}<.001 \\
.001\end{array}$ & $\begin{array}{l}3.52 \\
0.37\end{array}$ & .043 \\
\hline $\begin{array}{l}\text { REM-Latenz } \\
\text { 1. REM-Periode } \\
\text { 1. REM-Dichte } \\
\text { mittl. REM-Dichte }\end{array}$ & $\begin{array}{r}(\min ) 0.56 \\
(\min ) 0.48 \\
(\%) 2.92 \\
(\%) 1.70\end{array}$ & $\begin{array}{r}57.16 \\
4.80 \\
13.08 \\
0.92\end{array}$ & $\begin{array}{r}<.001 \\
.037 \\
.001\end{array}$ & $\begin{array}{r}10.77 \\
0.01 \\
5.96 \\
0.99\end{array}$ & $\begin{array}{r}<.001 \\
.007\end{array}$ \\
\hline
\end{tabular}

REM-Schlafes hingegen nahm zu. Gegenüber der Plazebo-Bedingung war die REM-Latenz deutlich verkürzt und die Dauer der 1. REM-Periode verlängert, bei gleichzeitig erhöhter REMDichte. Interaktions-Effekte (Gruppe $\mathrm{x}$,Treatment") konnten für die Dauer der Schlafperiode, die Einschlaf-Latenz, den Tiefschlaf, sowie für die REM-Latenz und die 1. REM-Dichte gefunden werden.

Um diese differentiellen Effekte von RS 86 genauer abklären zu können, wurde eine HauptkomponentenAnalyse durchgeführt (s. Tab. 3, oberer Teil). Bei den Depressiven war die Dauer der Schlafperiode nach Applikation von RS 86 tendenziell verkürzt, was auf eine signifikante Verlängerung der Einschlaf-Latenz zurückgeführt werden kann. Der Tiefschlaf war in der Gruppe der eßgestörten Patienten und bei den Kontrollen deutlich reduziert, nicht jedoch bei den Depressiven. Die Zunahme des REM-Schlafes konnte für die Kontrollen und die Depressiven statistisch abgesichert werden. In jeder der drei Stichproben kam es nach der Einnahme von RS 86 zu einer signifikanten Verkürzung der REM-Latenz. Die 1. REM-Dichte nahm jedoch nur bei den Depressiven signifikant zu.

Während der Plazebo-Nacht trennte lediglich die Dauer der Schlafperiode zwischen den Gruppen ( $F=3.49$, $\mathrm{df}=2, \mathrm{p}<0.05$ ). Nach der Applikation von RS 86 war die Einschlaf-Latenz der Depressiven im Vergleich zu der der eßgestörten Patienten und der Kontrollen deutlich verlängert $(F=3.49$, $\mathrm{df}=2, \mathrm{p}<0.01$ ). Die weiteren Parameter der Schlaf-Kontinuität und der Schlaf-Architektur differenzierten nicht zwischen den drei Gruppen. Die REM-Latenz der Depressiven war nun hochsignifikant kürzer $(\mathrm{F}=7.38, \mathrm{df}=2, \mathrm{p}<0.005)$ und die 1 . REM-Dichte höher $(F=5.72, \mathrm{df}=2, \mathrm{p}<0.01)$ als jene der $\mathrm{e} B-$ gestörten Patienten und der Kontrollen. Fünf der 7 Depressiven, jedoch nur eine eßgestörte Patientin mit Bulimia nervosa (18 Jahre, keine ,major depression“) zeigten eine sog. ,,sleep onset“" REM-Periode (REM-Latenz < 20 Minuten; vgl. Abb. 2).

Das Schlafmuster der eßgestörten Patienten mit einer ,"major depression" $(n=6)$ unterschied sich weder in der Plazebo- noch in der RS 86-Nacht von dem der nicht depressiven $E B s t o ̈ r u n g e n ~(n=6)$. Insbesonders war die mittlere REM-

\section{REM-Latenz}

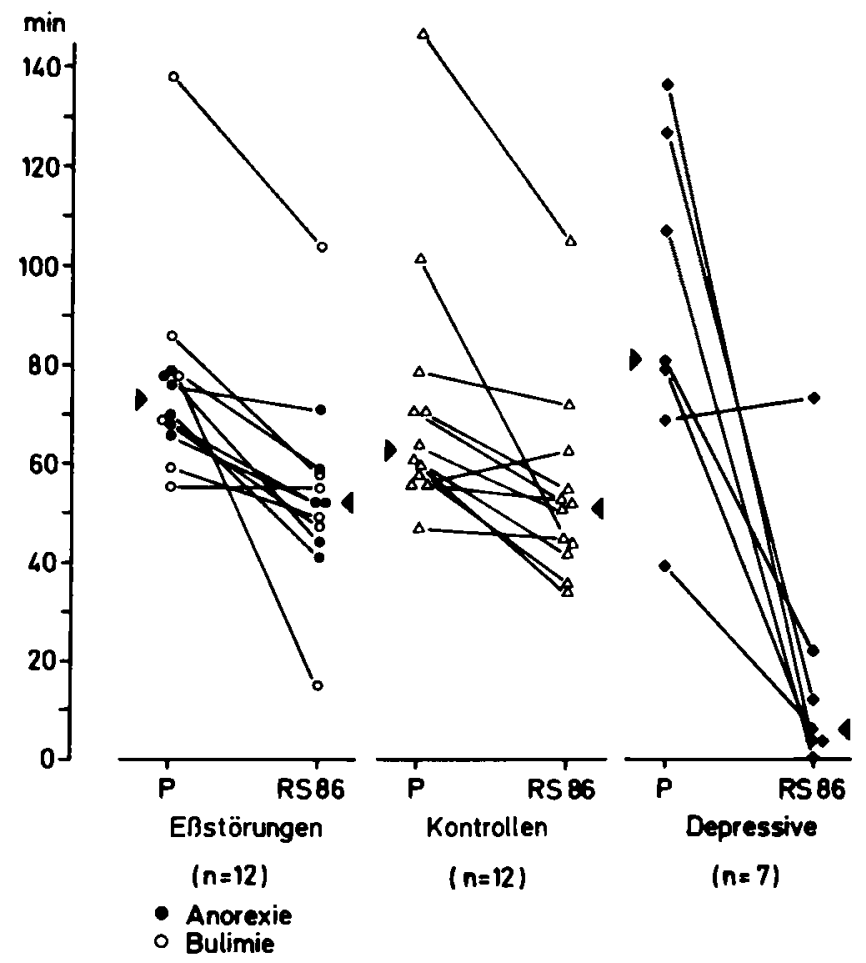

Abb. 2 REM-Latenz der Plazebo- und RS 86-Nacht. Die Pfeile markieren den jeweiligen Median. 
Latenz dieser beiden Gruppen jeweils vergleichbar (Placebo: $70 \pm 7 \mathrm{~min}$. vs $81 \pm 31 \mathrm{~min}, \mathrm{p}<0.65$; RS 86: $50 \pm 7 \mathrm{~min}$. vs $56 \pm 32 \mathrm{~min} ; \mathrm{p}<0.75$ ).

\section{Diskussion}

Insgesamt konnten keine Unterschiede zwischen dem Schlafmuster anorektischer und bulimischer Patienten, sowie dem normgewichtiger junger Gesunder beobachtet werden. Für die Bulimie-Patienten stehen die Resultate im Einklang mit den Berichten anderer Arbeitsgruppen (22, 33, 49). Die Vergleichbarkeit der Schlafstruktur anorektischer Patienten und gesunder Kontrollen scheint auf den ersten Blick in Widerspruch zu den Ergebnissen früherer Studien zu stehen, deren konsistenteste Beobachtungen eine fragmentierte Schlaf-Kontinuität und ein reduzierter Tiefschlaf waren $(10,31,33,38,49)$. $Z$ wischen diesen und der vorliegenden Untersuchung bestehen jedoch eine Reihe von methodischen Unterschieden, welche als Erklärung der diskrepanten Resultate angeführt werden können. Die Studien von Crisp et al. (10) und Lacey et al. (31) fokusierten den Zusammenhang von Gewichtzunahme und Veränderungen in der Schlafstruktur. In beiden Studien wurden keine Kontrollgruppen untersucht. Neil und Kollegen (38) dichotomisierten die untersuchten anorektischen Patienten nach einem normalen, bzw. pathologischen Wach-EEG und berichteten die Schlafvariablen lediglich von diesen beiden Untergruppen, nicht jedoch von der Gesamtstichprobe. Walsh et al. (49) analysierten die gemittelten Schlafdaten der ersten Nacht (Adaptationsnacht) und der zweiten Nacht, in welcher die Patienten und Kontrollen mit einem intravenösen Katheter schliefen. Beide Einflußgrößen - Adaptations- und Katheter-Effekt -- können den Schlaf nachhaltig im Sinne eines, ,arousal“"-Effektes beeinträchtigen $(1,6,24)$. In der vorliegenden Studie wurden, um Adaptationseffekte möglichst auszuschließen, lediglich die Polysomnogramme der jeweils 2., bzw. der 3. Nacht analysiert. Die beobachtete Unauffälligkeit der Schlaf-Kontinuität und Schlaf-Architektur der anorektischen Patienten stimmt mit den Befunden von Katz et al. (26) überein, welche ebenfalls nur die Schlafdaten der zweiten Nacht untersuchten. Auch die eher inkonsistent berichtete Verminderung des Tiefschlaf-Anteils kann auf solch ein artifiziell erhöhtes ,,arousal“-Niveau zurückgeführt werden. Hier sind allerdings noch weitere Einflußgrößen anzuführen. So berichteten Levy et al. (33), daß sie besonders bei jenen Anorexie-Patienten einen reduzierten Anteil des Tiefschlafes fanden, welche die Gewichtsreduktion durch striktes Einhalten von Diäten erreichten und keine intermittierenden Heißhunger-Attakken erlitten. Zum anderen konnten Phillips et al. (39) nachweisen, daß isokalorische Diäten mit unterschiedlicher Zusammensetzung der Makronährstoffe sich in verschiedener Weise auf den Tiefschlaf-Anteil auswirken. Diese möglichen Einflußgrößen wurden bei den früheren wie auch bei der vorliegenden Studie nicht kontrolliert.

Veränderungen des REM-Schlafes, besonders eine verkürzte REM-Latenz, sind ein häufig zu beobachtendes Phänomen bei depressiven Patienten und werden als charakteristische biologische Normabweichung der Depression angesehen (Übersicht bei 17, 29). Aus diesem Grunde wird die Beobachtung einer verkürzten REM-Latenz bei anorektischen und bulimischen Patienten als Hinweis auf eine biologische Verwandtschaft von Eßstörung und Depression gewertet $(26,38)$. Nach Dichotomisierung der untersuchten Patienten mit einer Eßstörung anhand der mittels eines standardisierten Instruments erhobenen Diagnose einer ,major depression“ wurden in der vor- liegenden Studie keine signifikanten Unterschiede zwischen den Schlafmuster von Eßstörungen mit und ohne einer Zusatzdiagnose einer Depression gefunden. Somit konnten die Resultate von Katz und Kollegen (26) und Walsh und Mitarbeiter (49), welche bei Eßstörungen mit depressiver Begleitsymptomatik verkürzte REM-Latenzen berichteten, nicht repliziert werden. $\mathrm{Zu}$ diesen Studien muß allerdings einschränkend angemerkt werden, daß es sich zum Einen um sehr kleine Stichproben handelte $(n=4 ; 49)$ und es zum Anderen bei der Untersuchung von Katz et al. (26) offen bleiben muß, ob es sich hierbei nicht um Patienten handelte, welche im Laufe ihrer Erkrankung das Vollbild eines depressiven Syndroms entwickelt hatten. Garfinkel und Garner (14) und auch $H s u(20)$ wiesen darauf hin, daß bei katamnestischen Untersuchungen solch ein Wandel im Krankheitsbild bei etwa $20 \%$ der anorektischen Patienten gefunden wurde. Im Hinblick auf die Untersuchung von Katz et al. (26) kann als ein Anhaltspunkt hierfür gewertet werden, daß die $\mathrm{Pa}$ tienten mit einer verkürzten REM-Latenz lediglich die ,lifetime"-Diagnose einer Anorexia nervosa erfüllten und ihre $\mathrm{Ha}$ milton-Depressions-Werte extrem hoch lagen. Die in der vorliegenden Studie gefundene Vergleichbarkeit der Schlafmuster von eßgestörten Patienten mit und ohne einer ,major depression“ bestätigt vielmehr die Ergebnisse von Hudson et al. (22) und Levy et al. (33).

Gegenüber den eßgestörten Patienten und den Kontrollen fand sich bei den jungen Depressiven lediglich eine erhöhte REM-Dichte; die REM-Latenz konnte wie auch in der Untersuchung von Hudson et al. (22) nicht zwischen diesen Gruppen trennen. Diese Beobachtung einer vergleichbaren REM-Latenz von Depressiven und gesunden Probanden scheint auf den ersten Blick im Widerspruch zu der Literatur zu stehen. Es existieren jedoch inzwischen einige Hinweise dafür, daß die für die Depression als typisch erachteten REM-Schlaf-Veränderungen altersabhängig sind $(15,30,48)$. In einer eigenen Untersuchung (32) konnte gezeigt werden, daß die REM-Dichte ab dem 25. Lebensjahr, die REM-Latenz erst ab Mitte des 4. Lebensjahrzehntes signifikant zwischen Depressiven und Gesunden trennten. Diese Befunde erklären die Vergleichbarkeit der REM-Latenz von jungen depressiven Patienten, gesunden Kontrollpersonen und auch von eßgestörten Patienten, wie sie in der vorliegenden und in der Studie von Hudson et al. (22) gefunden wurde.

Die Beobachtung, daß die Depressiven eine höhere REM-Dichte und auch häufiger verkürzte REM-Latenzen (bei nicht signifikant unterschiedlicher mittlerer REM-Latenz) aufwiesen als die Patienten mit einer Eßstörung und als die Kontrollen, kann als Hinweis darauf gewertet werden, daß das den REM-Schlaf regulierende System $(19,36)$ bei den jungen Depressiven bereits gestört ist; dies jedoch noch nicht in einem hinreichenden $\mathrm{Maße}$, um die Schlafstruktur nachhaltig zu beeinflussen.

Der REM-Schlaf-Induktions-Test mit dem Cholinomimetikum RS 86 führte bei den jungen Depressiven zu einer vollständigen Manifestation der REM-Schlaf-Dysregulation, welche vergleichbar mit der älterer Depressiver war (32). Sowohl bei den eßgestörten Patienten, als auch bei den Kontrollen konnte zwar ebenfalls ein REM-Schlaf-induzierender Effekt von RS 86 gesichert werden, dieser war jedoch hochsignifikant geringer ausgeprägt als bei den jungen Depressiven. Weiterhin konnte kein differenzieller EinfluB von RS 86 auf die Schlafstruktur depressiver und nicht depressiver EBstörungen festgestellt werden. 
Diese Resultate stehen nicht in Einklang mit den Beobachtungen von Sitaram und Kollegen (44), welche $66 \%$ der depressiven Anorexie-Patienten, jedoch nur $12 \%$ der nicht depressiven anorektischen Patienten als Responder auf Arecolin einstuften. Methodische Differenzen erschweren jedoch die unmittelbare Vergleichbarkeit dieser und der vorliegenden Studie. Sitaram et al. (44) infundierten Arecolin intravenös während der zweiten NonREM-Periode und klassifizierten die beobachtete Reaktion anhand eines Response-Kriteriums (Median der REM-Latenzverkürzung: 19.5 Minuten). Weiterhin hatten die depressiven Anorexie-Patienten zusätzlich die "life-time" Diagnose einer ,,major depression und/oder es lag eine familiäre affektive Erkrankung vor, was als ,trait"-Faktor interpretiert werden kann. In der vorliegenden Studie wies nur eine bulimische Patientin Hinweise für solch einen Faktor auf. Ihre REMLatenz lag jedoch sowohl in der Placebo-, als auch in der RS 86Nacht im Mittelbereich der Gesamtstichprobe. Die Bedeutung einer früheren Episode einer ,,major depression “ für die Reagibilität im RIT wurde kürzlich von Berger und Mitarbeitern (4) infragegestellt, da sie keinen differentiellen Effekt von RS 86 auf das Schlafmuster vollständig remittierter depressiver Patienten und gesunder Kontrollen fanden. Eine Einflußgröße, welche zumindest bei den anorektischen Patienten das Ergebnis des RIT mitbestimmt haben könnte, mag der Umstand sein, daß diese Patienten aus medizinischen Gründen erst nach einer Gewichtszunahme auf mindestens $80 \%$ ihres IBW an diesem Test teilnahmen. Sitaram et al. (44) stellten in ihrer Untersuchung jedoch keine unterschiedliche Häufigkeitsverteilung von Responder zwischen akut und remittiert anorektischen Patienten fest. Damit scheinen gewichtsbezogene Einflußgrößen auf das Ergebnis des RIT wenn, dann nur eine untergeordnete Rolle zu spielen.

Insgesamt sprechen die Ergebnisse der vorliegenden Studie dafür, daß bei jungen Depressiven eine Störung des den REM-Schlaf regulierenden Transmitter-Systems vorliegt, die jedoch noch nicht hinreichend ausgeprägt ist, um den Schlaf unter Baseline-Bedingungen in der Weise zu beeinträchtigen, wie es für ältere Depressive beschrieben ist. Durch die Applikation des cholinergen Stimulus RS 86 kann diese Störung jedoch demaskiert werden. Für die Patienten mit einer EBstörung konnten keine Hinweise für eine latent vorliegende Störung der zentralnervösen cholinerg-aminergen Transmitterbalance gefunden werden. Vielmehr konnten in dieser Gruppe die Effekte von RS 86 nicht von jenen unterschieden werden, welche bei den Kontrollen beobachtet wurden. Die dargestellten Resultate sprechen damit nicht für die Annahme einer biologischen Assoziation von EBstörung und Depression $(8,21,25)$, sondern vielmehr für das Modell, in welchem die bei anorektischen und bulimischen Patienten häufig zu beobachtende depressive Symptomatik als sekundäres Phänomen der Mangelernährung, bzw. der intermittierenden bulimischen Attacken und den daraus resultierenden Gefühlen der Insuffizienz angesehen wird $(7,9,12)$.

\section{Literatur}

1 Adams, $K$.: Sleep is changed by blood sampling through an indwelling venous catheter. Sleep 5 (1982) 154-158.

2 Berger, M.: REM-Schlaf und cholinerges System bei depressiven Erkrankungen. In: H. Hippius, E. Rüther, M. Schmauss (Hrsg.) SchlafWach-Funktionen. Springer-Verlag, Berlin-Heidelberg-New York (1987) $181-190$

3 Berger, $M$., et al.:Cholinomimetic drug RS 86, REM sleep, and depression. Lancet (1985) 1385-1386.
- Berger, M., et al.: The cholinergic REM sleep-induction-test with RS 86: State- or trait-marker of depression? Arch. Gen. Psychiatry (in Druck).

5 Binswanger, L.: Der Fall Elen West. Schweiz. Arch. Neurol. Psychiat. 54 (1944) 69-117.

- Browman, C.P., Cartwright, R.D.: The first night effect on sleep and dreams. Biol. Psychiatry 15 (1980) 809-812.

7 Bruch, H.: Eating disorders. Obesity, anorexia nervosa and the person within. Basic Book, New York 1973.

- Cantwell, D.P., et al.: Anorexia nervosa: An affective disorder? Arch. Gen. Psychiatry 34 (1977) 1087-1093.

- Crisp, A.H.: Anorexia nervosa: Let me be. Grune \& Stratton, New York 1980

10 Crisp, A.H., Stonehill, E., Fenton, G.W.: An aspect of the biological basis of the mind-body apparatus: The relationship between sleep, nutritional state and mood in disorders of weight. Psychother. Psychosom. 18 (1970) 161-175.

11 Degkwitz, R., et al.: Diagnoseschlüssel und Glossar psychiatrischer Krankheiten der WHO, ICD, 9. Revision. Springer Verlag, BerlinHeidelberg-New York 1980.

12 Fairburn, C.G.: Bulimia: its epidemiology and management. Psychiatr. Annals 13 (1983) 953-961.

13 Feighner, J.P., et al.: Diagnostic criteria for use in psychiatric research. Arch. Gen. Psychiatry 26 (1972) 57-63.

14 Garfinkel, P., Garner, D.: Anorexia nervosa: A multidimensional perspective. Brunner/Mazel, New York 1982.

is Gillin, J.C., et al.: Age-related changes in sleep in depressed and normal subjects. Psychiat. Res. 4 (1981) 73-78.

16 Gillin, J.C., Sitaram, N., Mendelson, W. B.: Acetylcholin, sleep, and depression. Hum. Neurobiol. 1 (1982) 211-219.

17 Gillin, J.C., et al.: Sleep and affective illness. In: R. M. Post, J.C. Ballenger (eds.) Neurobiology of Mood Disorders. Williams \& Wilkins, Baltimore (1984) 157-189

18 Halmi, K. A., et al.: Basic biological overview of the eating disorders. In: H. Y. Meltzer (ed.) Psychopharmacology: The Third Generation Of Progress. Raven Press, New York (1987) 1255-1266.

19 Hobson, J. A., Lydic, R., Baghdoyan, H. A.: Evolving concepts of sleep cycle generation: From brain centers to neuronal populations. Behav. Brain Science 9 (1986) $371-448$.

$20 \mathrm{Hsu}$, I.K.G.: Outcome of anorexia nervosa: A review of the literature. Arch. Gen. Psychiatry 37 (1980) 1041-1046.

21 Hudson, J.I., Laffer, P.S., Pope, H.G.: Bulimia related to affective disorder by family history and response to dexamethasone suppression test. Am. J. Psychiatry 139 (1982) 685-687.

22 Hudson, J.I., et al.: Sleep EEG in bulimia. Biol. Psychiatry 22 (1987) $820-828$

23 Janowsky, D.S., et al.: A cholinergic-adrenergic hypothesis of mania and depression. Lancet (1972) 632.

24 Jarrett, D.B., et al.: Effects of nocturnal intravenous cannulation upon sleep-EEG measures. Biol. Psychiatry 19 (1984) 1537-1550.

25 Katz, J.L.: Eating disorder and affective disorder: Relatives or merely chance aquaintances? Compr. Psychiatry 28 (1987) 220-228.

$20 \mathrm{Katz}, J . L$. et al.: Is there a relationship between eating disorder and affective disorder? New evidence from sleep recordings. Am. J. Psychiatry 141 (1984) 753-759.

27 Koehler, K., Sass, H.: Diagnostisches und Statistisches Manual Psychischer Störungen, DSM-III. Deutsche Bearbeitung und Einführung. Beltz-Verlag, Weinheim, Basel 1984.

28 Kraepelin, E.: Manic-depressive insanity and paranoia. Livingstone, Edinburgh 1921

29 Kupfer, D.J.: The sleep EEG in diagnosis and treatment of depression. In: A.J. Rush, K.Z. Altshuler (eds.) Depression. Basic mechanisms, Diagnosis, and Treatment. Guilford Press, New York (1986) 102-125.

30 Kupfer, D.J., et al.: EEG sleep, depression, and aging. Neurobiol. Aging 3 (1982) $351-360$.

31 Lacey, J.H., et al.: Weight gain and the sleeping electroencephalogram: Study of 10 patients with anorexia nervosa. Brit. Med. J. 4 (1975) 556-558.

32 Lauer, C., Riemann, D., Berger, M.: Age, REM sleep, and depression. Sleep Res. 16 (1987) 283.

33 Levy, A.B., Dixon, K.N., Schmidt, H.S.: REM and delta sleep in anorexia nervosa and bulimia. Psychiat. Res. 20 (1987) 189-197.

34 Lorr, $M$, et al.: Inpatient multidimensional psychiatric scale (IMPS). Consulting Psychologist Press, Palo Alto 1962. 
35 McCarley, R. W.: REM sleep and depression: Common neurobiological control mechanism. Am. J. Psychiatry 139 (1982) 565-570..

36 McCarley, R.W., Hobson, J.A. : Neuronal excitability modulation over the sleep cycle: A structural and mathematical model. Science 189 (1975) 58-60.

${ }^{37}$ Metropolitan Life Insurance Co. Stat. Bull. Met. Life Insur. Co. 40 (1959) $1-17$.

38 Neil. J.F., et al.: Waking and all-night sleep EEG's in anorexia nervosa. Clin. Electroencephal. 11 (1980) 9-15.

39 Phillips, F., et al.: Isocaloric diet changes and electroencephalographic sleep. Lancet 2 (1975) 723-725.

40 Rechtschaffen, A., Kales, A.: A manual of standardized terminology, techniques, and scoring system for sleep stages of human subjects. Superintendent of Documents, U.S. Government Printing Office, Washington D.C. 1968.

41 Riemann, D., et al.: The influence of the cholinergic agonist RS 86 on normal sleep with regard to gender and age. Psychiat. Res. 24 (1988) 137-144.

42 Russell, G.: Anorexia nervosa and bulimia nervosa: In: G. Russell, L. Hersov (eds.) Handbook of psychiatry, Vol. 4, The Neuroses and Personality Disorders. Cambridge University Press (1983) 285-298.

43 Semler, G., et al.: Test-retest reliability of a standardized psychiatric interview (DIS/CIDI). Eur. Arch. Psychiat. Neurol. Sci. 236 (1987) 214-222.
44 Sitaram, N., Gillin, J.C., Bunney, W.E.: Cholinergic and catecholaminergic receptor sensitivity in affective illness: Strategy and theory. In: R.M. Post, J.C. Ballenger (eds.) Neurobiology of Mood Disorders. Williams \& Witkins, Baltimore (1984) 629-651.

43 Spiegel, $R$.: Effects of RS 86, an oraly active cholinergic agonist, on sleep in man. Psychiat. Res. 11 (1984) 1-13.

40 Strober, M., Katz, J.L.: Do eating disorders and affective disorders share a common etiology? A dissenting opinion. Int. J. Eating Disorder 6 (1987) 171-180.

47 Swift, W.J., Andrews, D., Barklage, N.E.: The relationship between affective disorder and eating disorders: A review of the literature. Am. J. Psychiatry 143 (1986) 290-299.

48 Ulrich, R.F., Shaw, D. H., Kupfer, D.J.: Effects of aging on EEG sleep in depression. Sleep 3 (1980) 31-40.

49 Walsh, B.T., et al.: EEG-monitored sleep in anorexia nervosa and bulimia. Biol. Psychiatry 20 (1985) 947-956.

so Weilburg, J.B., et al.: Sleep architecture in bulimia: A pilot study. Biol. Psychiatry 20 (1985) 225-228.

\section{Dr.phil. Christoph Lauer}

Psychiatrische Universitätsklinik

Hauptstraße 5

7800 Freiburg 